\title{
The Role of Drifts in the Plasma Transport at the Tokamak Core-SOL Interface
}

\author{
A.V. Chankin* and D.P. Coster \\ Max-Planck-Institut für Pasmaphysik, EURATOM Association, 85748 Garching, Germany
}

\begin{abstract}
The interface between the core (inside the magnetic separatrix in X-point configurations) and the scrape-off layer (SOL) of tokamaks is a delicate region of the magnetic topology transition from closed to open field lines where neither the standard neoclassical theory nor the SOL physics fully apply. Sharp gradients of plasma parameters in the outer core, caused by the proximity of divertor sinks in the near SOL, invalidate some ordering assumptions of the neoclassical theory. At the same time, the existence of closed flux surfaces in the core enforces ambipolarity of radial plasma flows, in difference to the situation in the SOL where the current loop may close through the divertor. Detailed analysis of the plasma transport and flows with the emphasis on the outer core region, just inside the separatrix, is carried out in the paper, based on EDGE2D modelling and analytical formulas.
\end{abstract}

JNM keywords: Plasma-Materials Interaction, Plasma Properties

PSI-20 keywords: JET, EDGE2D, drifts, SOL transport

PACS: 52.55.Fa, 52.40.Hf, 52.65.-y, 52.25.Fi

*Corresponding author address: Max-Planck-Institut für Plasmaphysik, Boltzmannstr. 2, 85748 Garching, Germany

*Corresponding author E-mail: Alex.Chankin@ipp.mpg.de 
Presenting author: Dr A.V. Chankin

Presenting author E-mail: Alex.Chankin@ipp.mpg.de 


\section{Introduction}

This work was inspired by a pioneering work of Goldston [1] predicting the power width at the divertor target (Eqs. (5,6) of [1]) and particle confinement time $\tau_{\mathrm{p}}$ (Eq. (3)) for low-gas-puff, low density H-mode tokamaks. It is based on the assumption that the SOL density width is determined by the penetration length of ions through the separatrix by ion $\nabla \mathrm{B}$ and centrifugal (' $\nabla \mathrm{B}+$ cent.') drifts in the absence of strong anomalous particle transport. It also requires a rather low recycling rates which otherwise would influence the SOL density width. Further, the model assumes that the anomalous perpendicular electron heat conduction coefficient $\chi_{\mathrm{e}}$ is greater than the effective diffusion coefficient associated with the ion drift transport, $\chi_{e}>D_{i, \text { eff }}$, the condition likely to be fulfilled due to smallness of $D_{i, e f f}$. The ion transport thus sets the SOL density width which can be easily 'filled' by electron energy regardless of the particular electron heat transport mechanism. The drift-based model of [1] was shown to be in a good agreement with experimental scalings for the power width. In addition, the model was also used to estimate the maximum ion current that can be carried by $\nabla B+$ cent. drifts, $I_{l o s s} \sim 4 \pi a n_{\text {sep }} T_{\text {sep }} / B$, and the associated particle confinement time $\tau_{\mathrm{p}}$. This estimate requires a factor 2 ion pressure up-down asymmetry, which is possible provided there are some other non-ambipolar mechanisms apart from drifts, e.g. a stochastic layer around the separatrix. Cross-field drifts containing a radial component relevant to the present study are shown in Fig. 1. With $\mathrm{E} \times \mathrm{B}$ drifts being intrinsically ambipolar, electric charge can be carried across the flux surface only by $\nabla \mathrm{B}+$ cent. drifts, with the vertical guiding centre (g.c.) current density flux given (approximately, see below) by $2\left(p_{e}+p_{i}\right) / e B R$. The total charge outflow from the bottom half of the torus must be equal to the 
inflow from the top half, to satisfy quasineutrality. Hence, $\left(p_{e}+p_{i}\right)$ must be up-down symmetric, in the absence of other non-ambipolar mechanisms. The above estimate for $I_{\text {loss, }}$, if confirmed, would therefore indicate the presence of some other non-ambipolar mechanisms apart from drifts (Sec. 5). At the same time, the main result of [1]: the estimate of the SOL power width, is unaffected by possible other non-ambipolar mechanisms, as long as they influence mostly electrons but not the ions.

\section{EDGE2D modelling}

EDGE2D cases with drifts suffer from poor stability, which is influenced by the presence of impurities, details of the magnetic configuration, boundary parameters, grid resolution and some other factors. For this study, a low density pure deuterium EDGE2D case with drifts was run, with separatrix electron density $n_{e, s e p}=0.6 \times 10^{19} \mathrm{~m}^{-3}$, controlled by gas puff and recycling (see the setup of EDGE2D runs in [2]), with $3 \mathrm{MW}$ of input power in the ion and $2 \mathrm{MW}$ in electron channels, with $\mathrm{I}_{\mathrm{p}} / \mathrm{B}_{\mathrm{t}}=2 \mathrm{MA} / 2 \mathrm{~T}$, spatially constant radial transport coefficients $\mathrm{D}_{\perp}=0.5 \mathrm{~m}^{-2}$ and $\chi_{\mathrm{e}, \mathrm{i}}=1.5 \mathrm{~m}^{-2}$, and with the configuration of JET \#56723 (see Fig.2, also [2]), with 16 poloidal rings in the core and SOL. The ion $\nabla \mathrm{B}$ drift was down. For such a low $\mathrm{n}_{\mathrm{e}, \mathrm{sep}}$, temperatures $\mathrm{T}_{\mathrm{e}, \mathrm{i}}$ were almost constant on core flux surfaces, as intended, since poloidally constant temperatures greatly facilitate analysis of the code output. However, separatrix collisionalities were too low: $v_{i i}^{*}=0.4$, with ions being in the Plateau regime, and $v_{e i}^{*}=1.35$ - with marginally collisional electrons. Hence, the results of this study may only be qualitative and indicative of some important physics mechanisms. 


\section{Up-down asymmetries and radial plasma flows}

Outer midplane (OMP) profiles are shown in Fig. 3. At the separatrix, ion poloidal Larmor radius, $\rho_{i \theta}=1 \mathrm{~cm}$, is close to the decay length of the ion pressure, $\lambda_{p_{i}}=1.2 \mathrm{~cm}$, the situation often encountered in H-modes. Such a sharp ion pressure profile invalidates an assumption of the standard neoclassical theory which requires $\rho_{i \theta}<<\lambda$ (see. e.g. [3]), with $\lambda$ being the decay length of plasma parameters. This greatly increases ion parallel viscosity $\Pi_{i \|}$, plotted in Fig. 4 together with ion static pressure along the outermost core ring c01. In EDGE2D, poloidal distance is counted from the outer to the inner target.

The ion static pressure shows a significant in-out (high field side - low field side) asymmetry, which will be discussed later in Sec. 5 together with ion parallel flows that determine $\Pi_{i \|}$. At the same time, the pressure up-down asymmetry is small. The latter is a necessary condition for zeroing the net drift-related charge flow across the separatrix, as pointed out in the Introduction. Indeed, in the core, in the absence of other non-ambipolar mechanisms, radial drift flows of ions and electrons must be equal. For electrons, smallness of their net radial flow follows from their near Boltzmann distribution: $n_{e} \sim \exp \left(e \Phi / T_{e}\right)$, resulting (for $T_{e}(\theta)=$ const) in the cancellation between their radial diamagnetic flux $c T_{e} \partial n_{e} / \partial l_{\theta} / e B \sim-c n_{e} E_{\theta} / B$ and radial $\mathrm{E} \times \mathrm{B}$ flux $c n_{e} E_{\theta} / B$ locally, at each poloidal position regardless of the shape of the $n_{e}(\theta)$ profile. For ions, however, diamagnetic and $\mathrm{E} \times \mathrm{B}$ flows due to up-down pressure asymmetry add to each other, hence, $p_{i}$ must be fairly up-down symmetric to ensure the ambipolarity of the plasma flow, with the $p_{i}(\theta)$ up-down symmetry being achieved by the adjustment of the radial electric field $\mathrm{E}_{\mathrm{r}}$ 
driving compressionable poloidal $\mathrm{E} \times \mathrm{B}$ flow. A similar cancellation for electrons can be also obtained using g.c. drifts, by writing electron fluxes as $\Gamma_{\nabla B+c e n t .}=2 c p / e B R \times \partial R / \partial s_{\theta}$, $\Gamma_{E \times B}=-c n / B \times \partial \Phi / \partial s_{\theta}$, combining the two drifts into $\Gamma_{d r i f t}=c T / e B R^{2} \times \partial\left(n R^{2}\right) / \partial s_{\theta}$, and calculating the total flow across the flux surface: $\Gamma_{\text {total }}=\int_{s_{\theta}=0}^{2 \pi} \Gamma_{d r i f t} d s_{\theta} \times 2 \pi R=$ $\int_{s_{\theta}=0}^{2 \pi} 2 \pi c T / e B R \times \partial\left(n R^{2}\right) / \partial s_{\theta}=0$ due to $B R=\operatorname{const}(\theta)$. Hence, the near cancellation of the radial electron flow, to the degree $T_{e}$ is poloidally constant and $n_{e}$ is distributed according to the Boltzmann law, follows from the cancellation between integral $\nabla \mathrm{B}+$ cent. and $\mathrm{E} \times \mathrm{B}$ flows. In particular, any up-down density asymmetry, leading to the net ion flow due to $\nabla \mathrm{B}+$ cent. drifts, will be cancelled by the $\mathrm{E} \times \mathrm{B}$ flow in the R direction arising from vertical electric field (E-field).

In the strictest formulation in flux coordinates, surface-averaged flux of species $j$ can be obtained from the momentum balance equation (Eq. (2.15 of [3]), giving:

$$
\left\langle n_{j} \mathbf{u}_{j} \cdot \vec{\nabla} \Psi\right\rangle=-c / e_{j}\left\langle R^{2} \vec{\nabla} \xi \cdot\left(e_{j} n_{j} \mathbf{E}+\mathbf{F}_{j}\right\rangle+c / e_{j}\left\langle R^{2} \vec{\nabla} \xi \cdot \vec{\nabla} \vec{P}_{j}\right\rangle+c / e_{j} \partial / \partial t\left\langle n_{j} m_{j} R^{2} \vec{\nabla} \xi \cdot \mathbf{u}_{j}\right\rangle,\right.
$$

where the notations are standard, with $\psi$ being poloidal flux, $\vec{\nabla} \xi$ - unit vector in the toroidal direction divided by major radius R, $\vec{P}_{j}$ - pressure-stress tenzor, $\mathbf{F}_{j}$ - friction force, and $\langle\ldots\rangle$ denoting flux surface averaging defined by Eqs. (2.55-2.67) of [3]. For electrons, neglecting their small viscosity and classical diffusive flux caused by perpendicular friction force with ions, and for steady state conditions, one obtains the neoclassical flux: $\left\langle n_{e} \mathbf{u}_{e, n c} \cdot \vec{\nabla} \Psi\right\rangle=$ $-c\left\langle R^{2} \vec{\nabla} \xi \cdot\left(e n_{e} \mathbf{E}+\mathbf{F}_{e, \|}\right\rangle / e\right.$ (Eq. (2.95) of [3]). Neglecting also the (usually small) Ware pinch 
related to the toroidal E-field (the only component present it this equation, since $\vec{\nabla} \xi \cdot \mathbf{E}_{\perp}=0$ ), one arrives at a conclusion that the net radial electron flow is determined by the surface-averaged electron-ion friction force. This friction force arises due to the Pfirsch-Schlüter (P-S) current, and contributes to the poloidal E-field and associated $\mathrm{E} \times \mathrm{B}$ drift in the R-direction as shown in Fig. 1, giving the P-S diffusion. In the circular geometry with concentric flux surfaces and $r / R<<1$, the P-S diffusion coefficient is given by $D_{P-S}=1.2 q^{2}\left(T_{e}+T_{i}\right) v_{e i} /\left(m_{e} \omega_{e}^{2}\right)$ (Eq. (4.102) of [3], with k-coefficients in Table II on p. 267)). The ratio of the P-S flow $\left(D_{P-S} d n / d r \times 2 \pi a 2 \pi R\right)$ to the particle flow associated with $I_{\text {loss }} \sim 4 \pi a n_{\text {sep }} T_{\text {sep }} / B$, is $1.2 \pi q^{2}\left(1+T_{e} / T_{i}\right) v_{e i} / \omega_{e} \times R / \lambda_{n}$, where $\lambda_{n}$ - density decay length. For JET parameters at the separatrix with $\mathrm{R}=1.5 \mathrm{~m}, \lambda_{n}=1 \mathrm{~cm}$, safety factor $\mathrm{q}=3, \mathrm{~T}_{\mathrm{e}}=\mathrm{T}_{\mathrm{i}}, \mathrm{n}_{\mathrm{e}}=1 \times 10^{19} \mathrm{~m}^{-3}, \mathrm{~T}_{\mathrm{e}}=100 \mathrm{eV}, \mathrm{B}=3 \mathrm{~T}$, this ratio is equal to $1 / 80$. Hence, the typical drift flow through the separatrix must be by 2 orders of magnitude lower than the value calculated from the $\nabla \mathrm{B}+$ cent. drifts by assuming factor 2 up-down pressure asymmetry.

Owing to the large role played by parallel ion viscosity, a certain degree of up-down asymmetry in the ion static pressure on closed field lines is nevertheless possible. The quantity that has to exhibit the complete up-down symmetry should be related to the vertical guiding centre (g.c.) non-ambipolar drifts ( $\nabla \mathrm{B}+$ cent. drifts) for both ions and electrons. After simple transformations, to account for larger ion centrifugal drift velocity compared to the $\nabla \mathrm{B}$ drift velocity (for the same kinetic energy), and neglecting electron viscosity, one arrives at the (newly introduced) 'currrent pressure': $p_{\text {current }}=p_{i \text {,static }}+\Pi_{i \|} / 4+n_{i} m_{i} v_{i \|}^{2} / 2+p_{e}$. Here $\Pi_{i \|}$ - parallel ion viscosity excluding ‘dynamic pressure' $n_{i} m_{i} v_{i \|}^{2}$. Fig. 5 shows poloidal profiles of the 'current pressure' for the $1^{\text {st }}$ 
ring (c01) inside the separatrix, together with rings 4,7 and 10 further inward, and for the $1^{\text {st }}$ ring outside of the separatrix in the SOL and divertor (s01), together with rings 4, 7 and 10 further outward. The current pressure profiles look qualitatively similar to those of the ion static pressure or total static pressure $\left(p_{e}+p_{i}\right)$ ones due to the presence of the coefficient $1 / 4$ before viscosity and a relatively small contribution from dynamic pressure $n_{i} m_{i} v_{i \|}^{2}$ (except for positions close to the target in the divertor, where the Mach number is high). In the core, all profiles are up-down symmetric, while in the SOL a clear up-down asymmetry in favour of the bottom half of the torus is seen, as expected from the effect of the ion $\nabla \mathrm{B}+$ cent. drifts. Radial transport in the SOL is, generally, non-ambipolar, with the current loop closing through the divertor.

\section{Plasma flows and in-out asymmetries at the core edge}

An interesting feature of core current pressure profiles shown in Fig. 5, which is also seen on $n_{e}$ profiles, is a significant in-out asymmetry in favour of the inner (high field) side, with the surface variable part being $\sim 1 / 3$ of the surface average value, which is explained later in this section. Owing to a nearly Boltzmann distribution of electrons, this also implies a large in-out asymmetry in the electric potential $\Phi$. Fig. 6 shows poloidal profiles of $\Phi$ and the Mach number of the parallel ion flow on the $1^{\text {st }}$ ring inside the separatrix. The $\Phi$ in-out asymmetry implies a significant outward (along R) E-field generating downward $\mathrm{E} \times \mathrm{B}$ drift shown schematically in Fig. 1. In the SOL, ion $\nabla \mathrm{B}+$ cent. drifts down together with the poloidal $\mathrm{E} \times \mathrm{B}$ flow pump particles to the lower half of the tours, with the particle flow loop being closed by ion flows along field lines from bottom to top ('ion Pfirsch-Schlüter flows', see e.g. [4]). In Fig. 6, the same $\sin (\theta)$ shaped feature of the Mach number profile as in the SOL is seen, with surface variable ion parallel flows from top to bottom. Its origin is however different. It is entirely attributed to the 
large downward $\mathrm{E} \times \mathrm{B}$ drift, while the combination of the ion $\nabla \mathrm{B}+$ cent. drifts and poloidal $\mathrm{E} \times \mathrm{B}$ flow would contribute to the (smaller) parallel flow in the opposite direction (from top to bottom). This is due to the opposite signs of radial electric field $\mathrm{E}_{\mathrm{r}}$ in the core and in the SOL (so that the two contributions are added in the SOL but subtracted in the core) and the fact that the influence of the $\mathrm{E} \times \mathrm{B}$ flow outweighs that from the ion $\nabla \mathrm{B}+$ cent. drifts (or, using the fluid formulation, outweighs the influence of the ion diamagnetic flow). The large negative $E_{r}$ extracted from EDGE2D for the $1^{\text {st }}$ ring inside the separatrix can be expressed through gradients of plasma parameters by using a numerical coefficient (a fitting parameter) before the ion temperature gradient as follows: $e E_{r}=\nabla p_{i} / n_{e}+1.29 \nabla T_{i}$. Since this coefficient is positive, the poloidal E $\times \mathrm{B}$ flow is numerically larger than the ion diamagnetic flow and should therefore drive the P-S flow in the opposite direction to that seen in the SOL. But, due to the presence of large vertical $\mathrm{E} \times \mathrm{B}$ flow, as pointed out above, the parallel ion flow profile looks qualitatively similar to that seen in the SOL. The coefficient 1.29 lies in between the values 0.5 and 2.1 obtained for Plateau and P-S regimes, respectively, derived for the simplest circular configuration with concentric flux surfaces and $r / R<<1$, as follows from [6] for the expression for poloidal velocity (see Eqs. (68-69), and we neglect the effect of a small toroidal momentum; also note that the coefficient before $\nabla \mathrm{T}_{\mathrm{i}}$ here corresponds to $-k$ in [6]). This coefficient therefore is not inconsistent with the neoclassical theory for a near separatrix plasma of the analyzed EDGE2D case, which is on the border between the Pfirsch-Schlüter and Plateau regimes.

The surface-averaged parallel ion flow shown in Fig. 6 is in the co-current direction. This should be attributed to the perpendicular viscosity ( $2^{\text {nd }}$ term on the right hand side (RHS) of Eq. (1)) due to the presence of the co-current plasma rotation in the SOL (where such a flow is 
experimentally observed and calculated in the codes, see e.g. [5]). The mechanism of radial transport of poloidal velocity from the SOL to the core was discussed in $[6,7]$.

The origin of the large ion-out density asymmetry is attributed to the mechanism of a compressible poloidal plasma $\mathrm{E} \times \mathrm{B}$ flow which scales as $E_{r} / B \times 2 \pi R \propto R^{2}$. Under an extreme (and unphysical) assumption of a finite $\mathrm{E}_{\mathrm{r}}$ but $T_{i} \rightarrow 0$, this would lead to a factor 4 in-out density asymmetry for a machine with $r / R=1 / 3$. In reality, $\mathrm{T}_{\mathrm{i}}$ and $\mathrm{E}_{\mathrm{r}}$ are related to each other, and pressure tends to reach the equilibrium along field lines. The $\rho_{i \theta} \approx \lambda_{p_{i}}$ equality in this EDGE2D case achieved at the separatrix implies that poloidal $E \times B$ drift velocity $c E_{r} / B \sim c \nabla p_{i} / e n B \sim c T_{i} / e \lambda_{p_{i}} B$ is close to the poloidal projection of ion thermal velocity $\sqrt{T_{i} / m_{i}} \times B_{\theta} / B$. The poloidal $\mathrm{E} \times \mathrm{B}$ drift under such conditions can strongly perturb the pressure distribution over the flux surface via the transfer of parallel momentum in the diamagnetic direction, leading to a substantial ion-out pressure asymmetry in favour of the inner (high field) side.

\section{Possible influence of field line stochastization on plasma flows}

As pointed out earlier, arguments against the build up of large up-down pressure asymmetries, leading to the estimate $I_{\text {loss }} \sim 4 \pi a n_{\text {sep }} T_{\text {sep }}$ / B for the ion current crossing the separatrix, should be waved provided there is an additional non-ambipolar mechanism in this region such as field line stochastization. If electrons are allowed to escape through the separatrix by following stochastic field lines, then $E_{r}$ in the core becomes more positive compared to prescriptions of the neoclassical theory while ions can follow electrons via an entirely neoclassical mechanism. More 
positive, or less negative, $E_{\mathrm{r}}$ leads to the build-up of up-down pressure asymmetries and the net large radial flow of ions (but not electrons!) due to $\nabla \mathrm{B}+$ cent. drifts. The deviation from the neoclassical $E_{r}$ can be measured in the experiment. Further, radial ion flow creates the $\mathrm{j} \times \mathrm{B}$ force accelerating the plasma toroidally in the co-current direction (with no such force for electrons following stochastic field lines). The generation of toroidal momentum is described by equating the LHS of Eq. (1) to the last term on its RHS, with a possible contribution from viscosity, $2^{\text {nd }}$ term on the RHS (see also Eq. (6.58) of [3], where it is considered for transient processes, since neoclassical transport on long time scales is ambipolar). In the presence of stochastization the $\mathrm{j} \times \mathrm{B}$ force exerts a permanent toroidal torque on the plasma (see e.g. $[9,10]$ ). The resulting toroidal momentum is a measurable quantity (but requires measurements at different poloidal locations).

\section{Summary}

Poloidal distributions of plasma parameters around the separatrix position under the conditions of high temperatures and sharp radial gradients of density and temperatures were modelled with the 2D fluid code EDGE2D. Large in-out (high field side - low field side), but small up-down asymmetries of plasma density, pressure and electric potential just inside of the separatrix, follow from the code, in contrast with one of the assumptions of the heuristic model [1] which required a factor two up-down pressure asymmetry for the prediction of the ion loss current through the separatrix and the $\tau_{\mathrm{p}}$ estimate. Such an assumption can only be made if one assumes the presence of other non-ambipolar processes of radial plasma transport in addition to drifts, e.g. anomalous radial electron transport caused by stochastization of magnetic field lines. The contribution of such processes to the radial transport could be experimentally detected via 
measurements of radial electric field and toroidal momentum inside the separatrix, in addition to direct experimental validation of $\tau_{\mathrm{p}}$ estimates of [1] and possibly, up-down pressure asymmetries along the separatrix flux surface. Further EDGE2D modeling of the near separatrix region is envisaged, by running cases with increased density (and hence, collisionality), to cover the range of separatrix collisionalities encountered in experimental conditions.

\section{Acknowledgement}

Stimulating discussions with Prof. P.C.Stangeby and members of the JET TFE2 edge modeling group are acknowledged.

\section{References}

[1] R.J.Goldston, Nucl. Fusion 52 (2012) 013009.

[2] A.V.Chankin, D.P.Coster, G.Corrigan et al., Plasma Phys. Control. Fusion 51 (2009) 065022.

[3] F.L.Hinton and R.D.Hazeltine, Rev. Mod. Phys. 48 (1976) 239.

[4] N.Asakura, S.Sakurai, M.Shimada et al., Phys. Rev. Lett. 84 (2000) 3093.

[5] A.V.Chankin, J. Nucl. Mater. 241-243 (1997) 199.

[6] R.D.Hazeltine, Phys. Fluids 17 (1974) 961.

[7] V. Rozhansky, Plasma Phys. Control. Fusion 46 (2004) A1-A17

[8] P A Molchanov, V A Rozhansky, S P Voskoboynikov, et al., Plasma Phys. Control. Fusion 50 (2008) 115010

[9] A.V.Chankin and W.Kerner, Nucl. Fusion 36 (1996) 563.

[10] E.Kaveeva, V.Rozhansky and M.Tendler, Nucl. Fusion 48 (2008) 075003. 


\section{Figure captions}

Fig. 1. Drifts with the radial component, near the separatrix. Vertical $E \times B$ drift is discussed in Sec. 4.

Fig. 2. Magnetic equilibrium

Fig. 3. Outer midplane (OMP) profiles

Fig. 4. Poloidal profiles at $1^{\text {st }}$ ring inside separatrix

Fig. 5. 'Current pressure’ profiles.

Fig. 6. Profiles along $1^{\text {st }}$ ring inside separatrix. 


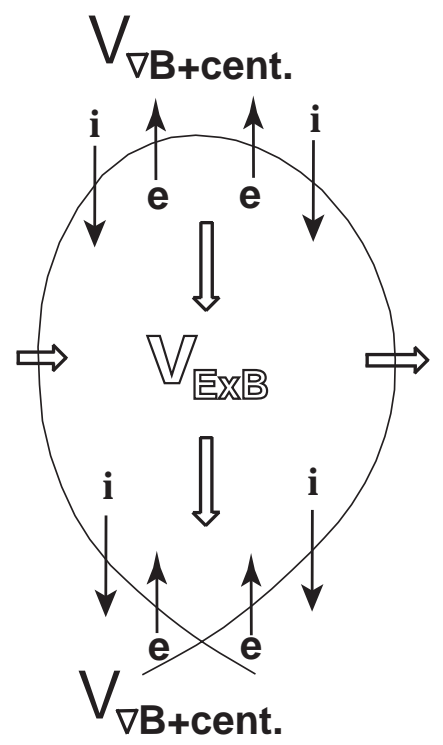

Fig. 1

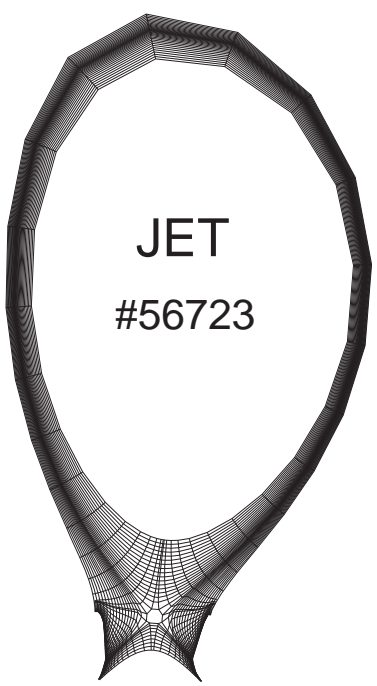

Fig. 2 

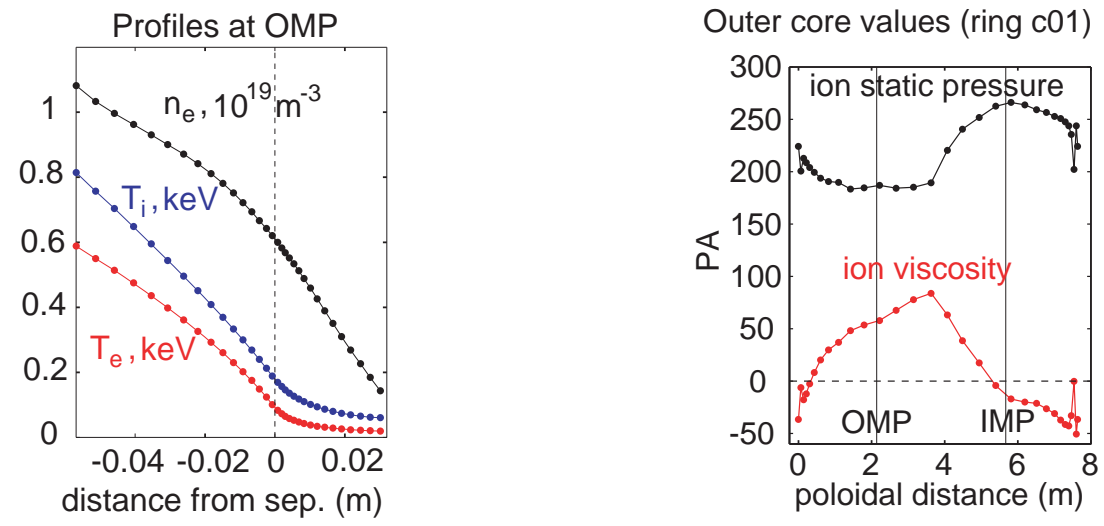

Fig. 3

Fig. 4 


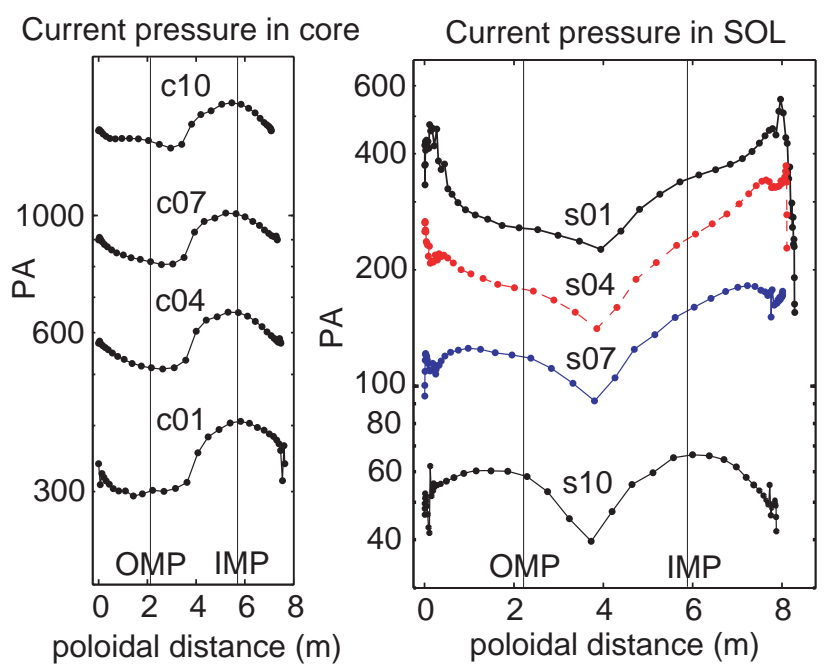

Fig. 5 


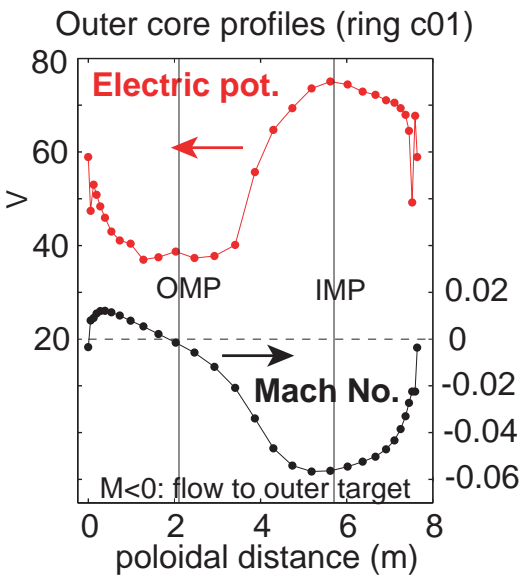

Fig. 6 